\title{
Factores determinantes de la productividad en las universidades públicas mexicanas
}

\section{Determining factors of productivity in Mexican public universities}

Teodoro Reyes Fongaa, Edgar Alfredo Nande Vázquez ${ }^{b}$, Lorena Hernández Ruiz ${ }^{\mathrm{c}}$

${ }^{a}$ Universidad de Colima, México. teodoro_reyesf@ucol.mx

${ }^{b}$ Universidad de Colima, México. cpnande@ucol.mx

c Universidad de Colima, México. lhernand@ucol.mx

\section{RESUMEN}

Dada la escasez de recursos las universidades públicas han puesto un especial interés en la consecución de sus objetivos de forma eficiente, lo anterior cobra una mayor relevancia ya que la Universidad es un organismo con metas no financieras, pero requiere de recursos públicos para poder operar. El objetivo de este estudio es estimar la productividad de las universidades públicas mexicanas durante el periodo 2007 a 2017, para ello, se utilizó la técnica no paramétrica del Análisis Envolvente de Datos para estimar los Índices de Productividad de Malmquist a un total de 44 universidades públicas. Los hallazgos principales indican que hay una evolución positiva en la productividad, la cual en promedio representa el $5,2 \%$ y que esta tiene principalmente una relación más significativa con la mejora de los procedimientos de investigación, y no necesariamente con el uso eficiente de los recursos de que disponen las unidades analizadas. Las conclusiones centrales son, que a partir de los Índices de Productividad de Malmquist se puede saber cuáles factores son los que contribuyen principalmente en la productividad, sin embargo, los esquemas de financiamiento para el contexto universitario mexicano podrían influir en la actuación de los gestores y en el resultado de estas.

Palabras clave: Productividad; Eficiencia; Índice de Productividad de Malmquist; Universidades públicas mexicanas

\section{ABSTRACT}

Given the scarcity of resources, public universities have placed a special interest in achieving their objectives efficiently, the above becomes more relevant because the university is an organization with non-financial goals, but it requires public resources to operate. The objective of this study is to estimate the productivity of the mexican public universities during the period 2007 to 2017, and for this, the nonparametric technique of the Data Envelopment Analysis was used to estimate the Malmquist Productivity Indexes to a total of 44 public universities. The main findings show that there is a positive evolution in productivity which, on average, represents $5,2 \%$ and that this has a more significant relationship with the improvement of research procedures, and not necessarily with the efficient use of resources of the units analyzed. The main conclusions are that, based on the Malmquist Productivity Indexes, it is possible to know which factors contribute mainly to productivity, however, the financing schemes for the Mexican University context could influence the performance of the managers and in their results.

Key words: Productivity; Efficiency; Malmquist productivity index; Mexican public universities 


\section{INTRODUCCIÓN}

En México hay 45 universidades públicas (tabla 1) que durante el periodo 2016-2017, atendieron a una matrícula de 1.652.235 estudiantes de educación superior lo que representa el 38,11\% del total nacional (UNAM, 2019).

En los últimos años las universidades públicas han crecido a un ritmo acelerado, debido al incremento en la demanda de los estudiantes, lo cual ha motivado la creación de nuevas unidades académicas para hacer frente a la demanda social en la educación; lo anterior establece un reto importante en la eficiencia de los procesos de las universidades públicas.

La política del gobierno federal de financiar a las universidades en función del número de alumnos provoca que los recursos que se reciben en las instituciones sean por debajo de la media nacional, lo cual ha generado la participación de las universidades en programas de recursos concursables para la atención de la expansión de los servicios educativos (Alcaraz \& Bernal, 2017).

Hoy más que nunca el sector público busca desarrollar sus actividades de forma más eficiente, lo cual se ha motivado por la dificultad de aumentar los ingresos públicos ordinarios y extraordinarios, y por la exigencia de mejores resultados sociales. Lo anterior tiene su fundamento en el paradigma de la Nueva Gestión Pública propuesto por Hood (1991) quien señala que el sector público debe actuar de manera eficiente y evaluar su desempeño; en este sentido, Fernández, Martínez y Fernández (2015) consideran que las universidades públicas deben rendir cuentas a la sociedad por el empleo de los recursos públicos y buscar alcanzar mejores niveles de productividad.

El objetivo de la presente investigación es doble; por una parte, se busca estimar la productividad de las universidades públicas de México, y desagregar los factores que la integran para conocer en qué medida se contribuye a ello, y por otra es ofrecer un panorama general de algunas técnicas utilizadas para estimar la eficiencia en las universidades.

En este trabajo se plantea como hipótesis que la productividad de las universidades públicas mexicanas está relacionada en mayor medida con las mejoras en sus procesos técnicos que en el uso eficiente de los recursos de que disponen.

Para alcanzar el objetivo que se proyecta, este trabajo se estructura como sigue; en el primer apartado se exponen aquellos aspectos que se deben considerar al momento de estimar la productividad de las universidades; después se hace una revisión de las evidencias empíricas que hay en la estimación de la eficiencia en el contexto universitario; seguidamente se presenta el diseño metodológico de la investigación; en el apartado de resultados, se presentan las estimaciones y se realiza la discusión de los mismos; y finalmente, se presentan las conclusiones alcanzadas.

Tabla 1. Universidades públicas de México.

\begin{tabular}{|l|r|}
\hline \multicolumn{1}{|c|}{ Universidad } & Matrícula \\
\hline Benemérita Universidad Autónoma de Puebla & 44.545 \\
\hline Centro de Investigación y de Estudios Avanzados del IPN & 1.844 \\
\hline Colegio de Posgraduados & 852 \\
\hline El Colegio de México & 331 \\
\hline Instituto Politécnico Nacional & 84.816 \\
\hline
\end{tabular}




\begin{tabular}{|c|c|}
\hline Instituto Tecnológico de Sonora & 14.735 \\
\hline Universidad Autónoma Agraria Antonio Narro & 4.071 \\
\hline Universidad Autónoma Benito Juárez de Oaxaca & 18.885 \\
\hline Universidad Autónoma Chapingo & 4.019 \\
\hline Universidad Autónoma de Aguascalientes & 11.660 \\
\hline Universidad Autónoma de Baja California & 35.067 \\
\hline Universidad Autónoma de Baja California Sur & 5.330 \\
\hline Universidad Autónoma de Campeche & 5.177 \\
\hline Universidad Autónoma de Chiapas & 18.486 \\
\hline Universidad Autónoma de Chihuahua & 21.124 \\
\hline Universidad Autónoma de Ciudad Juárez & 18.653 \\
\hline Universidad Autónoma de Coahuila & 21.458 \\
\hline Universidad Autónoma de Guerrero & 21.717 \\
\hline Universidad Autónoma de la Ciudad de México & 7.902 \\
\hline Universidad Autónoma de Nayarit & 10.684 \\
\hline Universidad Autónoma de Nuevo León & 66.400 \\
\hline Universidad Autónoma de Querétaro & 14.372 \\
\hline Universidad Autónoma de San Luis Potosí & 20.640 \\
\hline Universidad Autónoma de Sinaloa & 46.430 \\
\hline Universidad Autónoma de Tamaulipas & 39.353 \\
\hline Universidad Autónoma de Tlaxcala & 10.421 \\
\hline Universidad Autónoma de Yucatán & 11.237 \\
\hline Universidad Autónoma de Zacatecas & 15.000 \\
\hline Universidad Autónoma del Carmen & 3.983 \\
\hline Universidad Autónoma del Estado de Hidalgo & 19.760 \\
\hline Universidad Autónoma del Estado de México & 35.274 \\
\hline Universidad Autónoma del Estado de Morelos & 11.470 \\
\hline Universidad Autónoma Metropolitana & 46.418 \\
\hline Universidad de Colima & 10.883 \\
\hline Universidad de Guadalajara & 74.265 \\
\hline Universidad de Guanajuato & 13.188 \\
\hline Universidad de Quintana Roo & 3.179 \\
\hline Universidad de Sonora & 22.830 \\
\hline Universidad del Ejército y Fuerza Aérea & 1.621 \\
\hline Universidad Juárez Autónoma de Tabasco & 26.129 \\
\hline Universidad Juárez del Estado de Durango & 10.643 \\
\hline Universidad Michoacana de San Nicolás de Hidalgo & 36.135 \\
\hline Universidad Nacional Autónoma de México & 185.029 \\
\hline Universidad Pedagógica Nacional & 65.736 \\
\hline Universidad Veracruzana & 49.142 \\
\hline
\end{tabular}

Fuente: Elaboración propia con datos de la UNAM (2019).

\section{LA PRODUCTIVIDAD DE LAS UNIVERSIDADES PÚBLICAS EN MÉXICO}

La formación de capital humano altamente calificado sin duda alguna, es un factor determinante en el crecimiento económico de un país (Rodríguez, 2014), por 
tal motivo en México se han realizado numerosos esfuerzos para elevar el nivel de calidad de la educación en todos sus ámbitos. Lo anterior, ha derivado en diversas estrategias del Plan Nacional de Desarrollo, para elevar la calidad de la educación y han consistido en la evaluación de los programas educativos (López, Rodríguez, \& Ramírez, 2008).

En el sector público es muy difícil determinar una función de producción debido a la carencia de mercado y la carencia de precios en los productos académicos y de investigación (Martín, 2007); sin embargo, para el caso de la evaluación de la productividad del sector educativo en México es necesario adoptar el enfoque de Farrell (1957), quien estableció que una función de producción se determina a través de la relación existente entre los recursos productivos (inputs) y los productos esperados (outputs).

En los últimos años la Universidad ha crecido a un ritmo acelerado, debido al incremento de la tasa de estudiantes, lo cual ha motivado la creación de nuevas unidades académicas privadas para hacer frente a la demanda social en educación. Lo anterior, establece un reto importante para las universidades públicas en la formación de profesionales competitivos y responsables para la resolución de los problemas sociales.

Hoy ante un entorno de cambios continuos y de creciente desarrollo tecnológico, los procesos de gestión tradicionales de las universidades se hacen cada vez más obsoletos, razón por lo cual deben de apoyar sus acciones de creación de valor con herramientas estratégicas que les ayuden en la eliminación de los insumos innecesarios para incrementar la productividad, la reducción de los tiempos de respuesta en sus procesos y la flexibilidad de la gestión de sus organizaciones para integrar todos los recursos puestos a su disposición y alcanzar mejores niveles de productividad.

Tomando en consideración que en el contexto universitario existe una diversidad de actividades y de factores que intervienen en los procesos académicos, de investigación y de gestión, la estimación de la productividad se vuelve más compleja; algunos trabajos han identificado a los factores utilizados en el proceso universitario considerando como inputs al financiamiento y como outputs los procesos relacionados con la docencia y la investigación (Martín, 2007; Alcaraz \& Bernal, 2017).

El reto actual de la Universidad pública en México es la formación de profesionales competitivos en todos los ámbitos y además la generación de soluciones que ayuden a la sustentabilidad de las organizaciones y el cuidado del medio ambiente. Lo anterior hace más necesaria la evaluación y la medición de la actuación de las universidades públicas mexicanas.

Una herramienta desarrollada para evaluar el contexto educativo fue el Análisis Envolvente de Datos (DEA por sus siglas en inglés), el cual surgió a partir de la tesis doctoral de Rhodes (1978), quién se basó en el trabajo de Farrell de 1957 (Johnes, 2006), esta herramienta ha sido ampliamente utilizada para estudiar la eficiencia técnica de las unidades de producción, incluso más que las aplicaciones de fronteras estocásticas (Forsund \& Sarafoglou, 1999).

La técnica del DEA ha tenido un rápido desarrollo ya que en el periodo de 1978 a 2001 se tenían registradas 3.203 publicaciones que habían realizado investigaciones mediante la utilización de esta herramienta (Tavares, 2002). 
El DEA usa algoritmos de programación lineal para calcular la frontera, y su uso se ha popularizado por las ventajas que tiene, entre las que se encuentran: la facilidad de aplicarse en ámbitos con múltiples outputs, suministra información de organizaciones similares, no requiere especificar a priori una forma funcional para la tecnología, y no se necesita hacer supuestos de la distribución de los términos aleatorios (Coelli \& Perelman, 2001).

\section{EVIDENCIA EMPÍRICA}

La técnica de análisis DEA se ha utilizado en diversos estudios para estimar los índices de eficiencia de diversas universidades, por ejemplo McMillan y Datta (1998) estimaron la eficiencia relativa de 45 universidades en Canadá; Fernández, Martínez y Fernández (2015) calcularon la eficiencia técnica de 47 instituciones españolas; y por su parte, Quispe y Jordan (2017) realizaron una estimación de la eficiencia técnica de 11 organismos de sistema de educación superior de Bolivia.

En México, de igual manera existen trabajos que aplican el DEA para estimar la eficiencia de instituciones de educación (ver Tabla 2).

Tabla 2. Trabajos para estimar la eficiencia en instituciones de educación en México.

\begin{tabular}{|c|c|c|}
\hline Autores & Título & Enfoque \\
\hline Sigler (2004) & $\begin{array}{l}\text { Aplicación del } \\
\text { Data } \\
\text { Envelopment } \\
\text { Analysis a la } \\
\text { producción de } \\
\text { investigación } \\
\text { económica en la } \\
\text { Ciudad de México: } \\
\text { La eficiencia } \\
\text { relativa del CIDE, } \\
\text { COLMEX, IPN, } \\
\text { UAM y UNAM } \\
\text { (1990-2002) }\end{array}$ & $\begin{array}{l}\text { Se estima la eficiencia de los centros } \\
\text { públicos de investigación económica } \\
\text { durante el periodo de } 1990 \text { a } 2002 \text {, para } \\
\text { lo cual se emplea la técnica de análisis } \\
\text { DEA; las variables analizadas fueron } \\
\text { como inputs: investigadores adscritos } \\
\text { al Sistema Nacional de Investigadores } \\
\text { (SNI) y como outputs los artículos y } \\
\text { libros individuales. }\end{array}$ \\
\hline $\begin{array}{ll}\text { Altamirano } & \text { y } \\
\text { Peniche (2014a) }\end{array}$ & $\begin{array}{l}\text { Measuring the } \\
\text { Institutional } \\
\text { Efficiency Using } \\
\text { DEA and AHP: } \\
\text { The Case of a } \\
\text { Mexican } \\
\text { University }\end{array}$ & $\begin{array}{l}\text { Se estima la eficiencia de } 13 \\
\text { Dependencias de Educación Superior } \\
\text { de la Universidad Autónoma de } \\
\text { Querétaro, para ello se combinan las } \\
\text { técnicas de Proceso de Jerarquía } \\
\text { Analítica y el DEA; como intput se } \\
\text { utilizan: profesores con grado de doctor } \\
\text { y pertenecientes al SNI, y como } \\
\text { outputs: programas de estudio } \\
\text { pertenecientes al Padrón Nacional de } \\
\text { Posgrados de Calidad (PNPC), Cuerpos } \\
\text { Académicos y Programas Educativos } \\
\text { Acreditados. }\end{array}$ \\
\hline
\end{tabular}




\begin{tabular}{|c|c|c|}
\hline $\begin{array}{ll}\text { Altamirano } & y \\
\text { Peniche }(2014 b) & \end{array}$ & \begin{tabular}{lr}
\multicolumn{3}{l}{ Metodología AED- } \\
RNA para la \\
estimación de la \\
eficiencia \\
institucional: & \\
caso de las \\
dependencias de \\
educación \\
$\begin{array}{ll}\text { superior (DES) de } \\
\text { ingeniería } \\
\text { México de }\end{array}$
\end{tabular} & $\begin{array}{l}\text { Se lleva a cabo la estimación de } 51 \\
\text { escuelas de ingeniería de México con } \\
\text { una red neuronal artificial a partir de la } \\
\text { información generada con el DEA; } \\
\text { como variables inputs se utilizaron } \\
\text { habilitación de los Profesores de } \\
\text { Tiempo Completo (PTC) y como } \\
\text { outputs programas educativos de buena } \\
\text { calidad, programas reconocidos en el } \\
\text { PNPC y las brechas de competitividad } \\
\text { académica. }\end{array}$ \\
\hline $\begin{array}{l}\text { Navarro, Gómez y } \\
\text { Torres (2016) }\end{array}$ & $\begin{array}{lr}\text { Las universidades } \\
\text { en México: Una } \\
\text { medida de su } \\
\text { eficiencia a través } \\
\text { del análisis de la } \\
\text { envolvente } \\
\text { datos de } \\
\text { bootstrap }\end{array}$ & $\begin{array}{l}\text { Se hace una estimación de la eficiencia } \\
\text { de } 32 \text { universidades públicas en } \\
\text { México, con la aplicación del DEA y se } \\
\text { refuerza con la técnica de bootstrap, } \\
\text { identificando la eficiencia técnica } \\
\text { global, pura y de escala; para ello se } \\
\text { utilizan como input el financiamiento y } \\
\text { como outputs las actividades de } \\
\text { docencia e investigación. }\end{array}$ \\
\hline $\begin{array}{lll}\text { Alcaraz y } & \text { Bernal } \\
(2017) & & \end{array}$ & $\begin{array}{l}\text { Evaluación de la } \\
\text { eficiencia técnica } \\
\text { de las } \\
\text { universidades } \\
\text { públicas estatales } \\
\text { (UPE) del noroeste } \\
\text { de México } \\
\text { mediante Análsis } \\
\text { Envolvente de } \\
\text { Datos (DEA) }\end{array}$ & $\begin{array}{l}\text { Se estima la eficiencia técnica de las } \\
\text { universidades públicas estatales del } \\
\text { noroeste de México utilizando el DEA; } \\
\text { para ello se utilizan como input el } \\
\text { financiamiento federal y como outputs } \\
\text { alumnos, programas acreditados y } \\
\text { cuerpos académicos. }\end{array}$ \\
\hline
\end{tabular}

Fuente: elaboración propia.

\section{MetodologíA}

De las 45 universidades públicas mexicanas, solo se estimó la productividad de 44 durante el periodo de 2007 a 2017, ya que la Universidad Autónoma del Estado de Morelos presentó datos incompletos que imposibilitaban realizar las estimaciones correctamente, por lo que, para evitar sesgos en los resultados se decidió no considerarla en el estudio.

Para conocer el nivel de desempeño de las universidades analizadas, se empleó el Índice de Productividad Total de Malmquist (IPM), el cual se fundamenta en la estimación de las funciones de distancia; con el objetivo de identificar las contribuciones del cambio en eficiencia y cambio tecnológico en la productividad, Fare, Grosskopf, Lindgren, y Roos (1992) proponen la descomposición del Índice de Productividad Total de Malmquist.

La estimación de las funciones distancia del IPM, se realizó utilizando el método de programación lineal DEA el cual fue desarrollado por Charnes, Cooper y 
Rhodes (1978); el DEA es una técnica de programación matemática que estima una frontera envolvente empírica a partir de un conjunto de inputs y outputs del grupo de unidades analizadas (Coll \& Blasco, 2000).

Para realizar las estimaciones, Fare, Grosskopf, Norris y Zhang (1994), proponen estimar cuatro funciones de distancia que permitan medir el cambio del IPM en dos períodos. Para realizar el estudio se empleó el modelo DEA con rendimientos constantes conocido como modelo CCR, el cual considera a una entidad como eficiente cuando aumenta la cantidad de outputs manteniendo constante los recursos utilizados, y la orientación del modelo fue output.

El modelo con orientación output se describe de la siguiente manera:

$$
\operatorname{IPM}\left(y_{t}, x_{t}, y_{t+1}, x_{t+1}\right)=\frac{D_{o}^{t+1}\left(y_{t+1}, x_{t+1}\right)}{D_{o}^{t}\left(y_{t}, x_{t}\right)}\left[\frac{D_{o}^{t}\left(y_{t+1}, x_{t+1}\right)}{D_{o}^{t+1}\left(y_{t+1}, x_{t+1}\right)} \frac{D_{o}^{t}\left(y_{t}, x_{t}\right)}{D_{o}^{t+1}\left(y_{t}, x_{t}\right)}\right]^{1 / 2},
$$

Donde el cociente que está fuera de los corchetes mide el cambio en la eficiencia entre los períodos $t$ y $t+1$, siendo equivalente al cociente de la eficiencia técnica de Farrell en el período $t+1$ y en el período $t$; muestra los cambios en el tiempo o efecto catching-up ${ }^{1}$ de la eficiencia, dejando ver si la unidad evaluada se está acercando o alejando de la frontera eficiente. Este cambio en el nivel de eficiencia, es consecuencia de la capacidad que tienen las organizaciones de incorporar progreso técnico en la gestión de su proceso productivo. La parte restante del modelo es la medida del cambio tecnológico, representado por la media geométrica de la transformación tecnológica entre los períodos t y $t+1$.

$\mathrm{Si}$ una unidad muestra entre dos períodos $\mathrm{t}$ y $\mathrm{t}+1$ un aumento en su productividad, el IPM tomará un valor mayor a 1; si por el contrario, la unidad pierde productividad a lo largo del período, el IPM tendrá un valor inferior a 1. En cambio, si no se produce variación en la productividad el valor del IPM será 1. Sin embargo, es importante considerar que los componentes del IPM pueden tomar direcciones opuestas, por lo que cabe la posibilidad que mientras que uno aumenta el otro disminuya, y viceversa.

Las variables input utilizadas en el estudio fueron: el financiamiento y el personal perteneciente al Sistema Nacional de Investigadores; mientras que las variables output elegidas para realizar el análisis fueron: el número total de artículos publicados en el ISI y Scopus, patentes y revistas. Es decir, el estudio busca estimar la productividad en el rubro de la investigación en las universidades públicas en México.

Los datos se obtuvieron del portal EXECUM Estudio Comparativo de las universidades mexicanas de la Universidad Nacional Autónoma de México (UNAM). Para realizar las estimaciones se utilizó el programa Data Envelopment Analysis desarrollado por Tim Coelli en el Centro para el análisis de la eficiencia y la productividad del Departamento de Econometría dela Universidad de Nueva Inglaterra.

${ }^{1}$ Por efecto catching-up se entiende el cambio en eficiencia propia de las organizaciones con respecto a la frontera de posibilidades de producción, considerando aparte al cambio técnico que indica los cambios en innovación. 


\section{Resultados}

Las estimaciones realizadas mediante el Índice de Productividad de Malmquist, obtenidas para cada universidad pública se presentan en la Tabla 3.

Tabla 3. Índices de Productividad de Malmquist estimados por unidad de análisis

\begin{tabular}{|c|c|c|c|c|}
\hline Universidad & effch & techch & pech & sech \\
\hline $\begin{array}{l}\text { Benemérita } \\
\text { Universidad } \\
\text { Autónoma de } \\
\text { Puebla }\end{array}$ & 0,998 & 1,066 & 1,046 & 0,954 \\
\hline $\begin{array}{l}\text { Centro de } \\
\text { Investigación y de } \\
\text { Estudios Avanzados } \\
\text { del IPN } \\
\end{array}$ & 1,000 & 1,101 & 1,000 & 1,000 \\
\hline $\begin{array}{l}\text { Colegio de } \\
\text { Posgraduados }\end{array}$ & 1,071 & 1,129 & 1,011 & 1,059 \\
\hline $\begin{array}{l}\text { El Colegio de } \\
\text { México }\end{array}$ & 0,883 & 1,171 & 0,973 & 0,908 \\
\hline $\begin{array}{l}\text { Instituto Politécnico } \\
\text { Nacional }\end{array}$ & 1,028 & 1,150 & 1,127 & 0,913 \\
\hline $\begin{array}{l}\text { Instituto } \\
\text { Tecnológico de } \\
\text { Sonora }\end{array}$ & 0,936 & 1,180 & 1,004 & 0,933 \\
\hline $\begin{array}{l}\text { Universidad } \\
\text { Autónoma Agraria } \\
\text { Antonio Narro }\end{array}$ & 0,830 & 1,197 & 0,895 & 0,927 \\
\hline $\begin{array}{l}\text { Universidad } \\
\text { Autónoma Benito } \\
\text { Juárez de Oaxaca }\end{array}$ & 1,037 & 1,108 & 1,137 & 0,913 \\
\hline $\begin{array}{l}\text { Universidad } \\
\text { Autónoma } \\
\text { Chapingo }\end{array}$ & 0,999 & 1,002 & 0,974 & 1,026 \\
\hline $\begin{array}{l}\text { Universidad } \\
\text { Autónoma de } \\
\text { Aguascalientes }\end{array}$ & 1,022 & 1,155 & 1,086 & 0,941 \\
\hline $\begin{array}{l}\text { Universidad } \\
\text { Autónoma de Baja } \\
\text { California }\end{array}$ & 0,967 & 1,009 & 0,976 & 0,991 \\
\hline $\begin{array}{l}\text { Universidad } \\
\text { Autónoma de Baja } \\
\text { California Sur }\end{array}$ & 0,818 & 1,202 & 0,880 & 0,929 \\
\hline $\begin{array}{l}\text { Universidad } \\
\text { Autónoma de } \\
\text { Campeche }\end{array}$ & 0,832 & 1,205 & 0,893 & 0,932 \\
\hline
\end{tabular}




\begin{tabular}{|c|c|c|c|c|}
\hline $\begin{array}{l}\text { Universidad } \\
\text { Autónoma de } \\
\text { Chiapas } \\
\end{array}$ & 1,021 & 1,230 & 1,037 & 0,985 \\
\hline $\begin{array}{l}\text { Universidad } \\
\text { Autónoma de } \\
\text { Chihuahua }\end{array}$ & 0,983 & 1,237 & 0,980 & 1,003 \\
\hline $\begin{array}{l}\text { Universidad } \\
\text { Autónoma de } \\
\text { Ciudad Juárez }\end{array}$ & 1,014 & 1,138 & 1,014 & 1,000 \\
\hline $\begin{array}{l}\text { Universidad } \\
\text { Autónoma de } \\
\text { Coahuila }\end{array}$ & 1,002 & 1,037 & 0,971 & 1,031 \\
\hline $\begin{array}{l}\text { Universidad } \\
\text { Autónoma de } \\
\text { Guerrero }\end{array}$ & 1,027 & 1,079 & 1,034 & 0,993 \\
\hline $\begin{array}{l}\text { Universidad } \\
\text { Autónoma de la } \\
\text { Ciudad de México }\end{array}$ & 1,090 & 1,148 & 1,145 & 0,952 \\
\hline $\begin{array}{l}\text { Universidad } \\
\text { Autónoma de } \\
\text { Nayarit }\end{array}$ & 0,935 & 1,187 & 0,935 & 1,000 \\
\hline $\begin{array}{l}\text { Universidad } \\
\text { Autónoma de Nuevo } \\
\text { León }\end{array}$ & 0,980 & 1,109 & 1,017 & 0,964 \\
\hline $\begin{array}{l}\text { Universidad } \\
\text { Autónoma de } \\
\text { Querétaro }\end{array}$ & 1,056 & 1,146 & 1,021 & 1,035 \\
\hline $\begin{array}{l}\text { Universidad } \\
\text { Autónoma de San } \\
\text { Luis Potosí }\end{array}$ & 1,000 & 1,048 & 1,000 & 1,000 \\
\hline $\begin{array}{l}\text { Universidad } \\
\text { Autónoma de } \\
\text { Sinaloa }\end{array}$ & 0,935 & 0,993 & 0,990 & 0,944 \\
\hline $\begin{array}{l}\text { Universidad } \\
\text { Autónoma de } \\
\text { Tamaulipas } \\
\end{array}$ & 0,976 & 1,056 & 0,963 & 1,013 \\
\hline $\begin{array}{l}\text { Universidad } \\
\text { Autónoma de } \\
\text { Tlaxcala } \\
\end{array}$ & 0,815 & 1,207 & 0,919 & 0,887 \\
\hline $\begin{array}{l}\text { Universidad } \\
\text { Autónoma de } \\
\text { Yucatán }\end{array}$ & 1,040 & 1,048 & 1,000 & 1,040 \\
\hline $\begin{array}{l}\text { Universidad } \\
\text { Autónoma de } \\
\text { Zacatecas } \\
\end{array}$ & 1,015 & 0,993 & 1,010 & 1,005 \\
\hline
\end{tabular}




\begin{tabular}{|c|c|c|c|c|}
\hline $\begin{array}{l}\text { Universidad } \\
\text { Autónoma del } \\
\text { Carmen }\end{array}$ & 0,818 & 1,202 & 0,872 & 0,939 \\
\hline $\begin{array}{l}\text { Universidad } \\
\text { Autónoma del } \\
\text { Estado de Hidalgo }\end{array}$ & 0,977 & 0,985 & 0,977 & 1,000 \\
\hline $\begin{array}{l}\text { Universidad } \\
\text { Autónoma del } \\
\text { Estado de México }\end{array}$ & 0,975 & 0,961 & 0,979 & 0,996 \\
\hline $\begin{array}{l}\text { Universidad } \\
\text { Autónoma } \\
\text { Metropolitana } \\
\end{array}$ & 0,848 & 1,046 & 0,938 & 0,905 \\
\hline $\begin{array}{l}\text { Universidad de } \\
\text { Colima }\end{array}$ & 1,000 & 0,986 & 1,000 & 1,000 \\
\hline $\begin{array}{l}\text { Universidad de } \\
\text { Guadalajara }\end{array}$ & 0,966 & 1,111 & 1,000 & 0,966 \\
\hline $\begin{array}{l}\text { Universidad de } \\
\text { Guanajuato }\end{array}$ & 0,982 & 1,060 & 0,984 & 0,999 \\
\hline $\begin{array}{l}\text { Universidad de } \\
\text { Quintana Roo }\end{array}$ & 0,795 & 1,206 & 0,871 & 0,913 \\
\hline $\begin{array}{l}\text { Universidad de } \\
\text { Sonora }\end{array}$ & 0,990 & 0,971 & 0,991 & 0,999 \\
\hline $\begin{array}{l}\text { Universidad del } \\
\text { Ejército y Fuerza } \\
\text { Aérea }\end{array}$ & 0,786 & 1,103 & 0,715 & 1,099 \\
\hline $\begin{array}{l}\text { Universidad Juárez } \\
\text { Autónoma de } \\
\text { Tabasco }\end{array}$ & 1,046 & 1,124 & 1,026 & 1,020 \\
\hline $\begin{array}{l}\text { Universidad Juárez } \\
\text { del Estado de } \\
\text { Durango }\end{array}$ & 0,943 & 1,081 & 0,936 & 1,008 \\
\hline $\begin{array}{l}\text { Universidad } \\
\text { Michoacana de San } \\
\text { Nicolás de Hidalgo }\end{array}$ & 0,979 & 1,028 & 0,992 & 0,987 \\
\hline $\begin{array}{l}\text { Universidad } \\
\text { Nacional Autónoma } \\
\text { de México }\end{array}$ & 0,938 & 1,092 & 1,000 & 0,938 \\
\hline $\begin{array}{l}\text { Universidad } \\
\text { Pedagógica } \\
\text { Nacional } \\
\end{array}$ & 0,947 & 1,139 & 0,935 & 1,014 \\
\hline $\begin{array}{l}\text { Universidad } \\
\text { Veracruzana }\end{array}$ & 1,019 & 1,001 & 1,046 & 0,974 \\
\hline Promedio & 0,958 & 1,098 & 0,981 & 0,977 \\
\hline
\end{tabular}

Fuente: elaboración propia.

Las universidades públicas con cambio en el factor total de productividad (tfpch) superior a 1 se consideran que tienen un cambio positivo y por lo tanto 
"técnicamente productivas" en el periodo de análisis. De las 44 universidades públicas mexicanas, 31 presentan cambio positivo en el Factor Total de Productividad (FTP), el cual en promedio representa el 9,93\%, siendo las 5 mejores la Universidad Autónoma de Chiapas, la Universidad Autónoma de la Ciudad de México, la Universidad Autónoma de Chihuahua, la Universidad Autónoma de Querétaro y el Colegio de Posgraduados. En contraste, las universidades que tienen un cambio negativo en el FTP son solamente 13 del total, las cuales en promedio muestran un decremento del 4,54\%. Respecto del total de universidades analizadas, el resultado promedio del FTP representa un 5,2\% de incremento.

El Factor Total de Productividad se compone del cambio en eficiencia (effch) y del cambio tecnológico (techch), en este sentido, de igual manera se observa un incremento en el cambio tecnológico en un número considerable de universidades públicas, en este caso 38, las cuales en promedio muestran un aumento del 11,94\%, mientras que de las 44 universidades analizadas el incremento es del 9,8\%; sin embargo, el promedio del cambio en eficiencia muestra un decremento del $4,2 \%$. Por lo que se puede decir que la productividad de las universidades públicas mexicanas deriva principalmente por las mejoras en los procedimientos que continuamente siguen en el área de la investigación.

$\mathrm{Al}$ analizar la desagregación del cambio en eficiencia en sus componentes, el cambio en eficiencia pura (pech) y el cambio en eficiencia a escala (sech), se observa que éste, se debe en mayor medida en el uso eficiente de los recursos de que disponen, ya que el índice promedio de todas las universidades fue del 98,1\%, mientras que la eficiencia a escala representó un 97,7\%.

La evolución de la productividad estimada mediante el promedio por año, se presenta en la Tabla 4; de igual manera, se puede observar que, en la mayoría de los años analizados, la productividad de las universidades se presenta principalmente por el cambio tecnológico; mientras que el cambio en eficiencia, se debe al cambio en eficiencia pura, lo que sugiere un correcto uso de los insumos de los cuales disponen las universidades para llevar a cabo sus actividades de investigación.

Tabla 4. Índices de productividad de Malmquistestimados por el promedio por año.

\begin{tabular}{|l|r|r|r|r|r|}
\hline Año & effch & techch & pech & \multicolumn{1}{c|}{ sech } & tfpch \\
\hline $2007-2008$ & 0,500 & 2,405 & 0,804 & 0,622 & 1,202 \\
\hline $2008-2009$ & 2,361 & 0,426 & 1,404 & 1,681 & 1,006 \\
\hline $2009-2010$ & 0,393 & 2,718 & 0,654 & 0,601 & 1,069 \\
\hline $2010-2011$ & 2,104 & 0,508 & 1,290 & 1,632 & 1,068 \\
\hline $2011-2012$ & 0,957 & 1,076 & 0,893 & 1,072 & 1,030 \\
\hline $2012-2013$ & 0,830 & 1,465 & 0,926 & 0,896 & 1,216 \\
\hline $2013-2014$ & 1,191 & 0,721 & 1,133 & 1,052 & 0,859 \\
\hline $2014-2015$ & 0,964 & 1,292 & 1,040 & 0,927 & 1,245 \\
\hline $2015-2016$ & 0,803 & 1,318 & 0,919 & 0,873 & 1,058 \\
\hline $2016-2017$ & 0,915 & 0,931 & 0,969 & 0,945 & 0,852 \\
\hline Promedio & 0,958 & 1,098 & 0,981 & 0,977 & 1,052 \\
\hline
\end{tabular}

Fuente: elaboración propia.

Las estimaciones del Índice de Productividad de Malmquist también permite conocer la evolución a través de los años, lo cual, determina en qué momentos hubo 
incrementos o decrementos en la productividad, en función de todo el conjunto de unidades analizadas.

En relación con los resultados mostrados en la tabla anterior, las principales mejoras en productividad de las universidades se dieron durante los periodos del 2007 al 2008 con un incremento del 20,2\%, del 2012 al 2013 con un incremento del $21,6 \%$, y del 2014 al 2015 con un incremento del 24,5\%.

\section{DISCUSIÓN}

Hasta el momento no se han realizado estudios que estimen los factores determinantes de la productividad de las universidades públicas en México, por lo que esta investigación es la primera en enfocarse en la estimación de los factores determinantes de la productividad de las universidades públicas mexicanas y en qué medida contribuyen a ello, por lo que la importancia de los hallazgos podrían ser un marco de referencia para estudios futuros.

En algunos estudios (Alcaraz \& Bernal, 2017; Martín, 2007; Ayaviri \& Zamora, 2016; Quispe \& Jordan, 2017; Navarro, Gómez, \& Torres, 2016) que estiman la eficiencia y que han sido aplicados al ámbito de las universidades públicas, coinciden con las actividades de docencia e investigación como funciones sustantivas de los organismos citados, utilizando en algunos casos como variables input: profesores y fin anciamiento, y como variables output: matrícula, programas acreditados y publicaciones.

Un factor fundamental en los procesos de las universidades es la dicotomía entre las actividades de docencia e investigación, ya que ambas son los objetivos primordiales del quehacer social de estos organismos. Es importante tomar en cosideración que para identificar los factores determinantes de la productividad de las universiades públicas mexicanas, primero se estimaron los Índices de Productividad de Malmquist y posteriormente se analizaron a partir de la desagregación de los factores que la conforman.

En las universidades que mostraron mejores resultados en su productividad es evidente que sus procesos de docencia e investigación son más consolidados y maduros, puesto que el cambio tecnológico es el factor más significativo con relación al uso de sus recursos productivos. En este caso, las instituciones en su mayoría usan eficientemente los recursos pero principalmente desarrollan procesos con una orientación a los resultados de la investigación, lo que les permite alcanzar mejores índices de productividad dentro del grupo de comparación.

En el caso de las organizaciones que tienen una menor productividad, son poco más de una tercera parte las que llevan a cabo procesos consistentes y orientados a la investigación, ya que el resto tienen una combinación inadecuada en el uso de sus inputs y al parecer se encuentran en la búsqueda de economías de escala como parte de sus procesos; esto último parece que se da sin que hayan alcanzado aún el uso eficiente de sus recursos. Posiblemente este grupo de universidades se orientan en procesos múltiples académicos y de investigación, y no toman en consideración los aspectos relacionados con la minimización en el uso de los recursos productivos de que disponen, motivo por el cual presentan esos resultados.

Lo anterior permiten ver que las universidades públicas mexicanas que formaron parte del estudio basan sus actividades de investigación en el uso eficiente de sus recursos, pero principalmente en la mejora de sus procesos de investigación; 
por lo tanto, con base en la hipótesis que se ha planteado ésta se acepta, ya que en los resultados se puede observar la relación directa y significativa de la productividad en las universidades públicas mexicanas en relación con la mejora de sus procesos, y en menor medida con el uso eficiente de sus recursos.

Esta investigación representa un primer acercamiento a la estimación de los factores determinantes en la productividad de las universidades públicas en México, y por lo tanto, es un punto de apoyo para futuras investigaciones que incluso puedan hacer un estudio comparativo entre regiones de la evolución de la productividad en las universidades públicas de México o tal vez hacer una contrastación entre las universidades públicas y privadas para conocer cuales generan mayor productividad.

\section{CONCLUSIONES}

En función de los resultados presentados y en relación, al primer propósito del objetivo de esta investigación, se puede concluir que los Índices de Productividad de Malmquist permiten estimar la productividad y desagregarla en sus componentes como son el cambio en eficiencia y el cambio tecnológico, y el cambio en eficiencia a su vez, se puede desagregar en sus componentes, los cuales son el cambio en eficiencia pura y el cambio en eficiencia a escala. Lo anterior, hace de esta técnica una excelente herramienta, ya que ofrece la posibilidad de conocer cuáles factores son los que contribuyen principalmente en el cambio de la productividad de las unidades analizadas.

Respecto al segundo propósito, de la revisión de la literatura se encontró que la técnica del Análisis Envolvente de Datos, es una herramienta que se utiliza ampliamente para realizar estimaciones de eficiencia en el contexto universitario y que además, se aplica combinada con otras como por ejemplo la técnica de proceso de jerarquía analítica, redes neuronales artificiales o técnicas de bootstrap.

La utilización de las variables incorporadas en el estudio como inputs: financiamiento y personal perteneciente al Sistema Nacional de Investigadores, y como outputs: número total de artículos publicados en el ISI y Scopus, patentes y revistas; proporciona información valiosa respecto al desempeño de la actividad de investigación de las universidades públicas de México.

Las universidades públicas mexicanas tienen procesos académicos y de investigación significativos, sin embargo, algunos casos en particular tienen una orientación principal hacia el uso de los recursos productivos que a la productividad en investigación, estos factores permiten suponer, que posiblemente los mejores índices están vinculados a organizaciones más experimentales, y que en el caso de aquellas que tuvieron índices menos significativos, se basan en procesos menos experimentales y más orientados hacia la docencia, o que tal vez requieren de mayor tiempo para poder adaptarse a los procesos de cambio y, al uso de técnicas específicas y particulares de cada institución para poder mejorar sus resultados.

Finalmente, es importante considerar que los resultados podrían tener intervenciones en relación con los programas de financiamiento, lo cual condicionaría las actuaciones de los gestores de las universidades públicas, con el fin de actuar en función de lograr metas concretas, y ello podría influir en los resultados de la productividad de las universidades del grupo de análisis. 


\section{BIBLIOGRAFÍA}

Alcaraz, D., \& Bernal, D. (2017). Evaluación de la eficiencia técnica de las universidades públicas estatales (UPE) del noroeste de México mediante Análsis Envolvente de Datos (DEA). Nova Scientia, 9(2), 393-410.

Altamirano, A., \& Peniche, R. (2014a). Measuring the Institutional Efficiency Using DEA and AHP: The Case of a Mexican University. Journal of Applied Research and Technology, 12, 63-71.

Altamirano, A., \& Peniche, R. (2014). Metodología AED-RNA para la estimación de la eficiencia institucional: El caso de las dependencias de educación superior (DES) de ingeniería de México. Nova Scientia, 6(2), 356-378.

Ayaviri, V., \& Zamora, G. (2016). Medición de la eficiencia en las Universidades. Una propuesta metodológica. Perspectivas(37), 7-22.

Charnes, A., Cooper, W., \& Rhodes, E. (1978). Measuring the efficiency of decision making units. European journal of operational research, 2(6), 429-444.

Coll, V., \& Blasco, O. (2000). Evaluación de la eficiencia mediante el análisis envolvente de datos. Valencia, España: Universidad de Valencia.

Coelli, T., \& Perelman, S. (2001). Medición de la eficiencia técnica en contextos multiproducto. En A. Alvarez, La medición de la eficiencia y la productividad (págs. 113-135). Madrid, España: Pirámide.

Fare, R., Grosskopf, S., Lindgren, B., \& Roos, P. (1992). Productivity changes in Swedish Pharmacies 1980-89. A nonparametric Malmquist Approach. Journal of Productivity Analysis, 13(1-2), 85-101.

Fare, R., Grosskopf, S., Norris, M., \& Zhang, Z. (1994). Productivity Growth, Technical Progress, and Efficiency Change in Industrialized Countries. The American Economic Review, 84(1), 66-83.

Farrell, M. (1957). The measurement of productive efficiency. Journal of The Royal Statistical Society, 120(3), 253-290.

Fernández, Y., Martínez, A., \& Fernández, J. (2015). Determinantes de la eficiencia en las universidades públicas españolas: Un análisis DEA en dos etapas. Investigaciones de economía de la educación, 10, 491-508.

Forsund, F., \& Sarafoglou, N. (1999). The Diffusion of Research on Productive Efficiency: The Economist's Guide to DEA Evolution. Discussion Paper 99-02, Department of Economics and Social Sciences, NLH.

Hood, C. (1991). A public management for all seasons? Public Administration, 69(1), 3-19.

Johnes, J. (2006). Measuring teaching efficiency in higher education: An application of data envelopment analysis to economics graduates from UK Universities 1993. European Journal of Operational Research, 174(1), 443-456.

López, A., Rodríguez, A., \& Ramírez, L. (2008). Eficiencia terminal en la educación superior: La necesidad de un nuevo paradigma. Revista de la Educación Superior, XXXVII(146), 135-151.

McMillan, M., \& Datta, D. (1998). The Relative Efficiencies of Canadian Universities: A DEA Perspective. Canadian Public Policy, 24(4), 485-511.

Martín, R. (2007). La eficiencia productiva en el ámbito universitario: Aspectos claves para su evaluación. Estudios de Economía Aplicada, 25(3), 793-811. 
Navarro, J., Gómez, R., \& Torres, Z. (2016). Las universidades en México: Una medida de su eficiencia a través del análisis de la envolvente de datos con bootstrap. Acta universitaria, 26(6), 60-69.

Quispe, G., \& Jordan, W. (2017). Medición de la Eficiencia Técnica en las Universidades Autónomas del Sistema Universitario Boliviano: Aplicación del Análisis Envolvente de Datos (DEA). Espacios, 38(45), 3-23.

Rhodes, E. (1978). Data Envelopment Analysis and Approaches for Measuring the Efficiency of Decision-making Units with an Application to Program FollowThrough in U.S. Education. Ph. D. dissertation, School of Urban and Public Affairs Carnegie-Mellon University.

Rodríguez, R. (2014). Educación superior y transiciones políticas en México. Revista de la Educación Superior, XLIII(171), 9-36.

Sigler, L. (2004). Aplicación del Data Envelopment Analysis a la producción de investigación económica en la Ciudad de México: La eficiencia relativa del CIDE, COLMEX, IPN, UAM y UNAM (1990-2002). Birmingham: 4th International Symposium of Data Envelopment Analysis and Performance Management.

Tavares, G. (2002). A Bibliography of Data Envelopment Analysis (1978-2001). New Jersey, Estados Unidos de América: Rutgers University.

UNAM. (23 de enero de 2019). execum: explorador de datos 2017. Obtenido de Estudio comparativo de las Universidades Mexicanas: http://www.execum.unam.mx/ 